\title{
Structural pre-conceptual design studies for an EU DEMO equatorial EC port plug and its port integration
}

\author{
Peter Spaeh $^{\mathrm{a}^{*}}$, Christian Bachmann ${ }^{\mathrm{b}}$, René Chavan ${ }^{\mathrm{c}}$, Aljaz Cufar ${ }^{\mathrm{d}}$, Thomas Franke ${ }^{\mathrm{b}, \mathrm{e}}$, \\ Dirk Strauss ${ }^{\mathrm{a}}$, Minh Quang Tran ${ }^{\mathrm{c}}$ \\ ${ }^{a}$ Karlsruhe Institute of Technology, P.O. Box 3640, D-76021 Karlsruhe, Germany \\ ${ }^{b}$ EUROfusion Power Plant Physics and technology (PPPT) department, Garching, Germany \\ ${ }^{c}$ Swiss Plasma Centre EPFL, CH-1015 Lausanne, Switzerland \\ ${ }^{d}$ Reactor physics department, Jožef Stefan Institute, SI-1000 Ljubljana, Slovenia \\ ${ }^{e}$ Max-Planck-Institut für Plasmaphysik, Garching, Germany \\ *Corresponding author : peter.spaeh@kit.edu
}

For the EU DEMO Tokamak, Electron Cyclotron (EC) launching systems for plasma heating and stabilization are under development. Various concepts for the optical system are currently studied of which the Mid Steering Antenna (MSA) with a steering mirror at a recessed position behind the breeding blanket (BB), the Open Ended Waveguide (OEWG) concept with quasi-optical beam propagation by fixed mirrors only and the Remote Steering Antenna (RSA), currently seen as backup solution, are the basic ones. In addition, hybrid solutions, which means a port plug with different launcher concepts, are taken into consideration. In parallel, design drafts for a generic equatorial port plug are sketched with the aim to provide a versatile structural system, which allows customized installation of the potential optical systems. This paper presents a preconceptual hybrid design of an equatorial EU DEMO EC port plug based on MSA for plasma stabilization and OEWG for plasma heating, taking into account the exact port position with respect to the toroidal field coil, mechanical integrity, heat dissipation, neutronic shielding requirements, design integration and maintenance concepts.

Keywords: DEMO, ECRH, EC Launcher, Port Plug, Plasma Heating

\section{Introduction}

For the EU DEMO Tokamak, EC launching systems for plasma heating and stabilization are under development. Based on the 2017 engineering baseline, the 2018 physics baseline, and the port plugs being radially recessed to the level of the Vacuum Vessel (VV) inner shell and hence not blocking the vertical extraction of the outboard BB segments [1], a pre-conceptual design study of an EU DEMO equatorial EC launcher has been made. For optimum performance [2], the design study features a hybrid concept with MSA and also OEWG antennas. The MSA is considered for plasma stabilization and has a (partially) quasi-optical beam layout with steerable mirrors at a recessed position behind the radial extension of the breeding blankets [3]. Beside the MSA, also an OEWG antenna is designed for the EC Launcher, which will be used for plasma heating only.

The pre-conceptual design of the EC Launcher features structural components, which can accommodate this hybrid millimeter-wave system accordingly. It also comprises shielding elements, cooling installations and interface provisions for the integration of the optical system. All structural components were designed with respect to available design requirements on position and dimensions, mechanical and thermal loads, nuclear constraints, physics performance, maintenance and geometrical and functional interfaces.

The CAD model is set up by parametric skeleton geometries, which guarantees a relatively flexible design to cope with moving targets and potential future baseline changes (e.g. the concept of an RSA).

\section{DEMO Engineering baseline 2017}

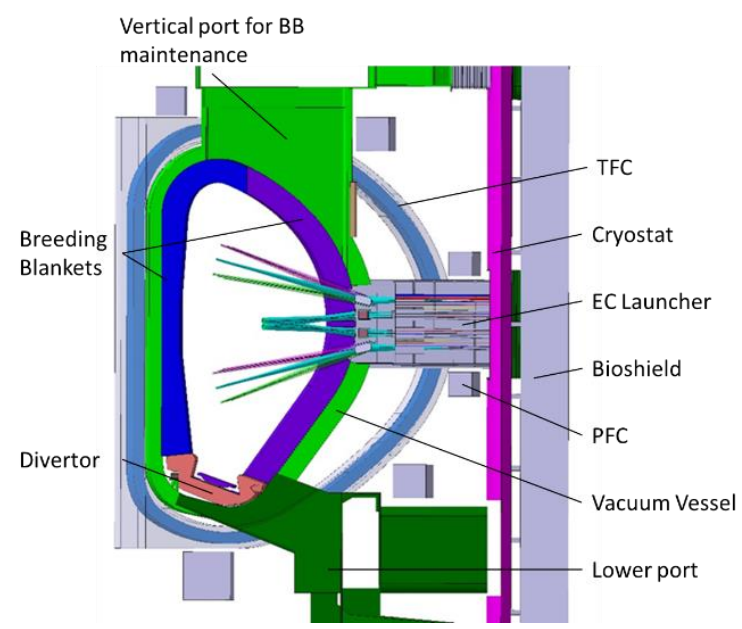

Fig. 1. EU DEMO baseline model 2017 and EC launcher 
Figure 1 outlines a cross-section of the EU DEMO engineering baseline model 2017 [4], having installed the EC launcher already. This model provides the current layout of the EU DEMO Tokamak, including the breeding blankets, the vacuum vessel with its vertical upper ports, the bioshield, the cryostat and many more interfacing and design-relevant components of an EC Launcher for a future EU DEMO reactor.

\section{EC Launcher design}

Beside the engineering baseline model 2017, the EC launcher design is made according to the physics baseline model 2018 and the latest optical system design, provided by the Italian Institute for Plasma Science and Technology (ISTP) [2], (cf. figure 2).

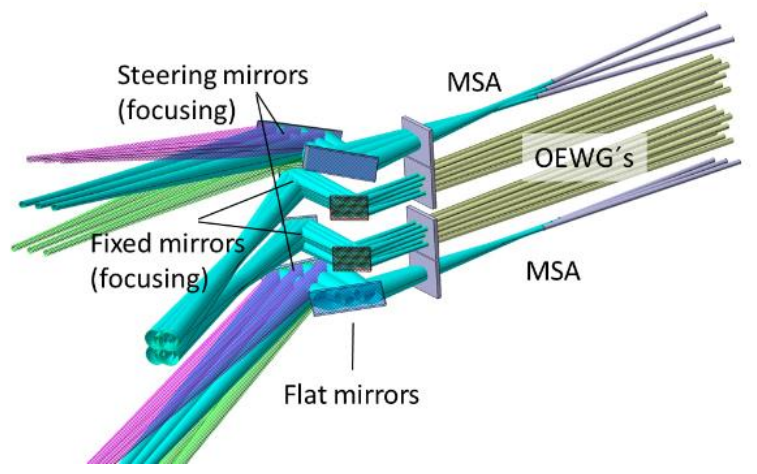

Fig. 2. Current EC Launcher millimeter wave system

It is comprised of an MSA with two sections of three beamlines each for NTM (Neo-classical Tearing Modes) control and two further OEWG antenna sections with eight waveguides each for plasma heating. Both systems are arranged in a symmetric layout with respect to the poloidal mid-plane of the EC launcher, such that the heating section is located in the inner area and the stabilization section is located on top and bottom, respectively.
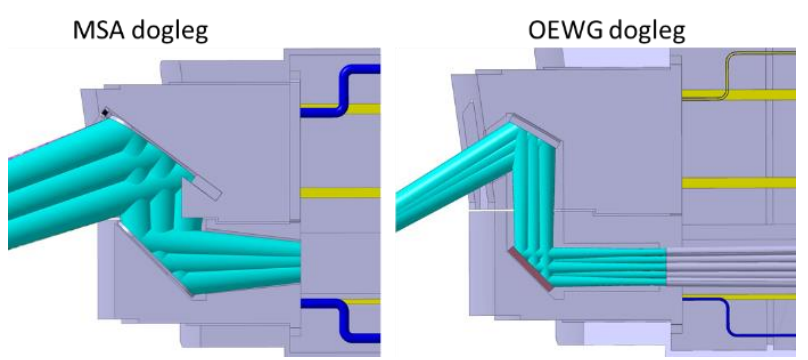

Fig. 3. Dogleg layout of the quasi-optical sections of the EC Launcher

From the tips of the waveguides, the microwave power is propagated as quasi-optical beams through the front part of the EC launcher. There both the MSA systems and the OEWG antennas are arranged with a dogleg layout (cf. figure 3), where flat mirrors reflect the beams from the side where the waveguides are located towards the other side, where focusing mirrors inject then the beams through openings in the breeding blanket into the plasma. All relevant parameters of the optical system can be found in [2]. Figure 4 gives an overview on the integration of the EC launcher into the engineering baseline model.

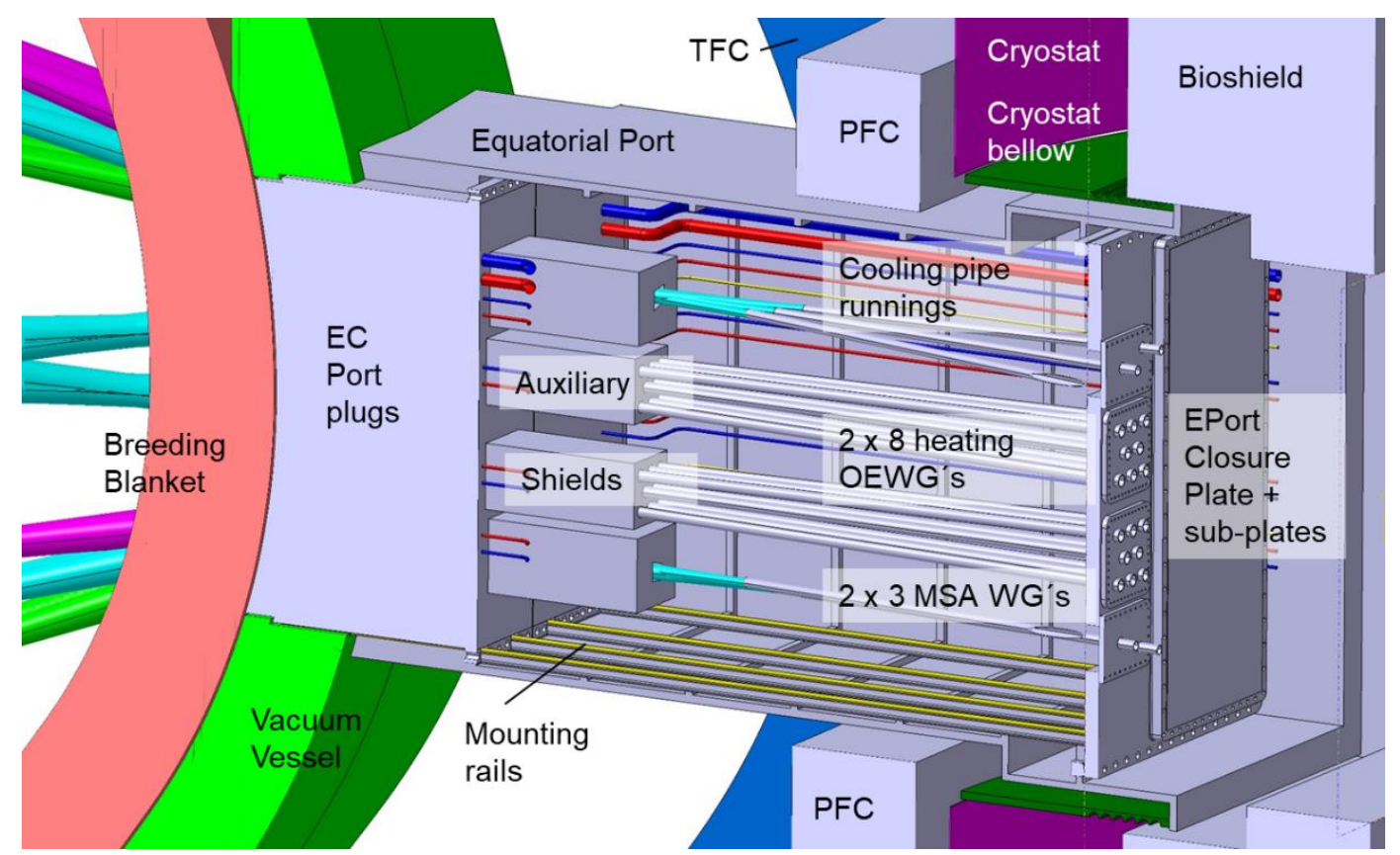

Fig. 4. EU DEMO EC H\&CD Launcher (cutaway)

\section{Equatorial Port for the EC launcher}

The equatorial ports in the current EU DEMO concept offer the possibility of customized dimensions for particular installations. The dimensional limits are set on principle by the poloidal clearance between the poloidal field coils and the toroidal clearance between the toroidal field coils only while the maximum radial extension is defined by the position of the Bioshield.

This means in fact a maximum height of the equatorial port of $4,000 \mathrm{~mm}$ and a maximum width of around $4,700 \mathrm{~mm}$. The greatest possible length is in the range of $6,300 \mathrm{~mm}$. The basic design of DEMO equatorial ports 
features a cantilevered structure, extruded from the vacuum vessel towards the Bioshield. The port design must enable proper accommodation of all installations required for the In-Vessel-section of the EC launching system. It also shall provide nuclear shielding both by itself and by suitable in-port shield equipment.

Since the port will experience volumetric heat from neutrons and photons and possibly plasma radiation as well, it shall be prepared with an adequate internal cooling system (which most likely will be the cooling system of the Vacuum vessel itself). From the mechanical point of view, it has to be designed with respect to DEMO operational loads, its own dead weight, payloads and operation under vacuum conditions. Provisions for smooth maintenance procedures shall complete the design of the EC equatorial port.

The EC port consists of three major segments - the vessel extrusion, the central port section and the rear end. The vacuum vessel extrusion has a wall thickness of $200 \mathrm{~mm}$ and forms the shell into which the EC launcher port plugs will be mounted. This section is expected to be actively cooled. For neutronic reasons the volumetric ratio between steel and water shall be in the range of $60 / 40 \%$ through $80 / 20 \%$. From this front element, the port then is stretched by the central port section. This part is formed by a rectangular cask where the In-vessel waveguides, the cooling lines and potential auxiliary shielding is located. Behind the central section, the profile of the port is further broadened in order to accommodate closure plates, which allow well-controlled removal of the port plugs for maintenance as well as additional space for the sidestepped layout of the coolant and Helium supply lines. A poloidal cutaway of the port is shown in Figure 5.

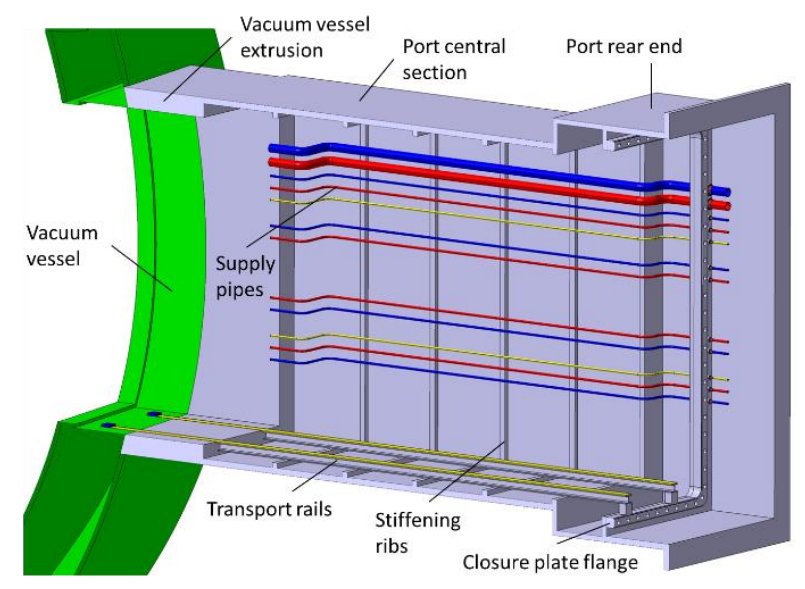

Fig. 5. EC Launcher equatorial port

Proper integration of the current millimeter wave system leads to a height of $3,680 \mathrm{~mm}$ for the front and middle section of the port. The width is $2,630 \mathrm{~mm}$ for the vessel extrusion and $2,850 \mathrm{~mm}$ for the central port. The largest profile of the port is at its rear end section, where the maximum height is $4,180 \mathrm{~mm}$ while the width ranges up to $3,350 \mathrm{~mm}$. This pre-conceptual configuration results in a total mass of the port structure of around 55 to 60 tons.
The large hollow structure of the port raises high demands on its mechanical stiffness. Currently no dedicated load specifications for the EC Port are available which is why a very coarse approach for checking the thickness of the port's central section has been made. One of the critical load scenarios for this rectangular hollow structure is an ingress of coolant accident for which a maximum pressure of $0.2 \mathrm{MPa}$ applied to the port walls is assumed. Despite the side wall of the port does not perfectly match the constraints of a rigidly fixed plate, it is used here to get an estimation of the maximum stresses expected. The maximum bending stress $\sigma_{b, \max }$ at the long edges of a rigidly fixed plate can be calculated [5] according to equation (1),

$$
\sigma_{b, \max }=\frac{\beta \cdot q \cdot b^{2}}{t^{2}}
$$

where the geometrical factor $\beta$ is 0.3834 , the applied pressure is $\mathrm{q}=0.2 \mathrm{MPa}$, the height of the wall is $\mathrm{b}=3680 \mathrm{~mm}$ and the thickness of the wall is $\mathrm{t}=60 \mathrm{~mm}$. With these parameters we get $\sigma_{b, \max }=288 \mathrm{MPa}$. For the pre-conceptual design the material for the port extension is supposed to be stainless steel $316 \mathrm{~L}(\mathrm{~N})$, whose (unirradiated) yield strength at DEMO operation temperature of $40{ }^{\circ} \mathrm{C}$ [6] is in the range of $200 \mathrm{MPa}$. Thus the need for additional stiffening of the port walls by ribs is indicated. These ribs are sketched in the CAD model of the port on a conceptual level only. They require strength analysis after all relevant load cases are defined.

For maintenance reasons the bottom of the port is geared with rails, which are furnished with a thick Mo sprayed layer for low friction shunting of the port plugs. The rails are designed as $\mathrm{H}$-beams with a height of $80 \mathrm{~mm}$ in the present configuration. Since the mass of the port plugs is expected to be in the range of up to 60 tons, their integration into the port has to be made carefully.

\section{EC launcher port plug design}

The mirrors of the In-Vessel millimeter wave system require precise and safe installation into the DEMO equatorial port. Thus they are mounted into dedicated port plugs, which are basically massive structural components with customized shapes and cut-outs which guarantee undisturbed beam propagation into the plasma, maximum neutron shielding properties and straightforward maintenance processes. Due to their position close to the plasma also active cooling of the plugs will be required.

One single port plug, having installed all the eight Invessel mirrors and filling up the entire front section of the port would be the optimum design with respect to shielding capability and mirror alignment, but has some disadvantages regarding various $\mathrm{RH}$ classifications expected for the steering mirrors and the fixed mirrors, respectively. Also the mass of such a full-size port plug will be more than 70 tons, which makes any manipulation by maintenance robots extremely challenging.

Thus a concept with two separated port plugs has been established, where the port plug on the right hand side (looking towards the plasma) carries the mirrors which 
reflect the beams finally into the plasma, while the port plug on the left hand side features the plain mirrors, which provide the dog-leg configuration of the beam layout. The consequences of this staged port plug layout for the mirror alignment (and thus the need for additional adjustment mechanisms) will require future analysis, however.

\subsection{Steering mirror equatorial port plug}

A Steering mirror equatorial port plug (SM EPP) has been designed on a pre-conceptual level, featuring remarkable cut-outs for mirror installation and beam propagation and also a basic rear flange for solid fastening into the equatorial port. The Steering mirrors will be installed from grooves on top and bottom side of the port plug while the fixed mirrors for the heating beams will be inserted through the side-wall openings. Manufacturing considerations indicate the fabrication of such a port plug preferably from a massive forged block, which is subsequently machined into the final shape. But also alternative fabrication methods as HIP or a welded assembly or even additive manufacturing processes are conceivable. An isometric view of the SM EPP is given in figure 6 .

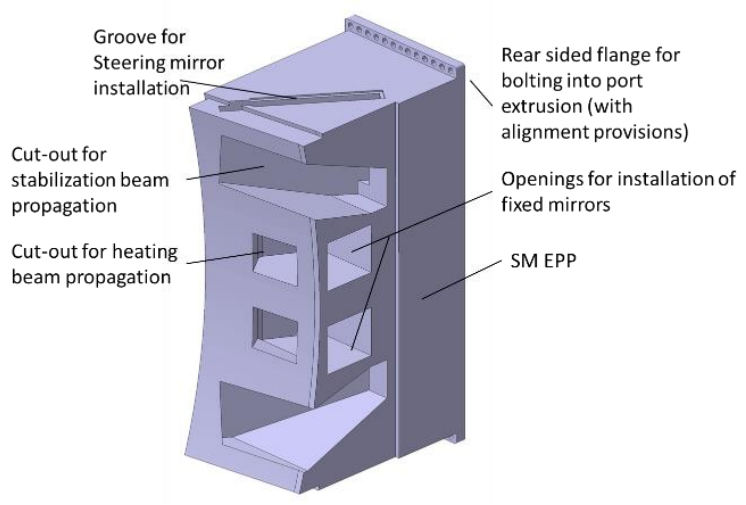

Fig. 6. EC Launcher SM EPP (full view)

The SM EPP has an outer dimension of approximately $3,240 \times 1,260 \times 2,000 \mathrm{~mm}^{3}$. In this conceptual design phase the nominal gaps between the port and the port plugs are $20 \mathrm{~mm}$ which shall cover manufacturing and assembly tolerances as well as space for acceptable deformation of the components during plasma disruptions. The outer profile of the plug provides various steps of $40 \mathrm{~mm}$ dimension, which in combination with the shape of the port create labyrinths with an overlap of $20 \mathrm{~mm}$ in order to minimize neutron streaming towards the rear.

The SM EPP will be equipped with a complex cooling system, which guarantees proper heat dissipation from all structural parts as well as dedicated cooling feed and return for all integrated millimeter wave components (i.e. the mirrors). For neutronic reasons the volumetric ratio between steel and water for the plug shall be adjusted between $60 / 40 \%$ and $80 / 20 \%$. The steering mirrors are expected to require regular exchange due to neutron damage. Since in-situ replacement of the mirrors only is not possible with this design, the whole port plug has to be taken out for this task. To make this process feasible, two skids will be mounted at the port plug's bottom side, which will provide the sliding contact between the port plug and the port during installation and maintenance procedures. The skids are made from a material with low friction properties like $\mathrm{Al}-\mathrm{Cu}$ alloys and are additionally coated with a DLC (diamond-like-carbon) layer. The total mass of the SM port plug is expected to be around 40 tons.

For the steering mirrors first fixation investigations have been made. The steering mirrors are mounted towards an individual mirror holder which then will be inserted precisely into the opening inside the plug.

Due to the much bigger size of the steering mirror and the higher neutron damage rates in DEMO compared to ITER, the use of the ITER steering drive is not applicable. This is why a new steering drive is under development currently, which is considered to be placed behind the mirror itself in order to benefit from those shielding capacity. For the present pre-conceptual design the drive was not sketched apart from the mirror bearings, which are realized by flexure pivots, which allow friction less movement of the steering mirror. However, due to the size of the mirrors, mechanical loads might turn out to be too high for these bearings and thus the feasibility of this concept requires further analysis. The basic assembly of the mirror holder and its integration is shown in figure 7.

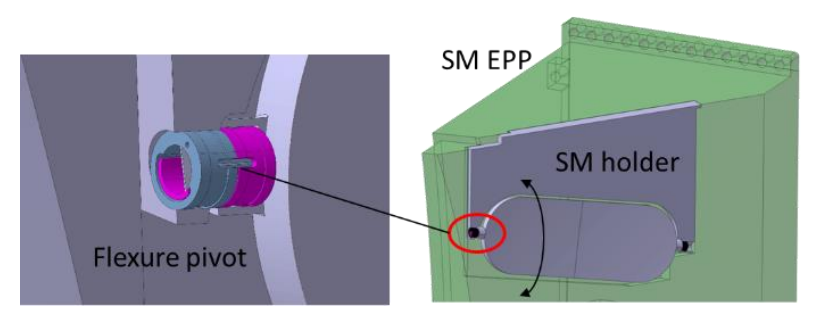

Fig. 7. Steering mirror holder integration

\subsection{Fixed mirror equatorial port plug}

For installation on the front left side of the equatorial plug for the EC launcher the fixed mirror equatorial port plug has been designed. It features a similar design approach as made for the SM EPP - a massive block with dedicated cut-outs for the mirrors and regarding beam propagation and a rear flange for aligned and reliable fastening inside the port. In addition the FM EPP has also loose bearings for the In-Vessel waveguides of the plasma heating section of the millimeter wave system. It also requires an active cooling system, removing nuclear heat and supplying cooling water to the mirrors installed.

However the FM EPP is expected to be designed for lifetime (due to its much better protection from the plasma by being located behind the breeding blanket), rails and skids are installed in order to allow repair or replacement in case of failure of the mirrors or damage of the plug itself.

The FM EPP has an outer dimension of approximately $3,240 \times 950 \times 2,000 \mathrm{~mm}^{3}$. As well as the SM EPP its outer shape creates labyrinth steps to block neutron streaming. Its total mass sums up to 27 tons for the basic component. 
Figure 8 shows the plug in an isometric view and with a cutaway through the OEWG section.
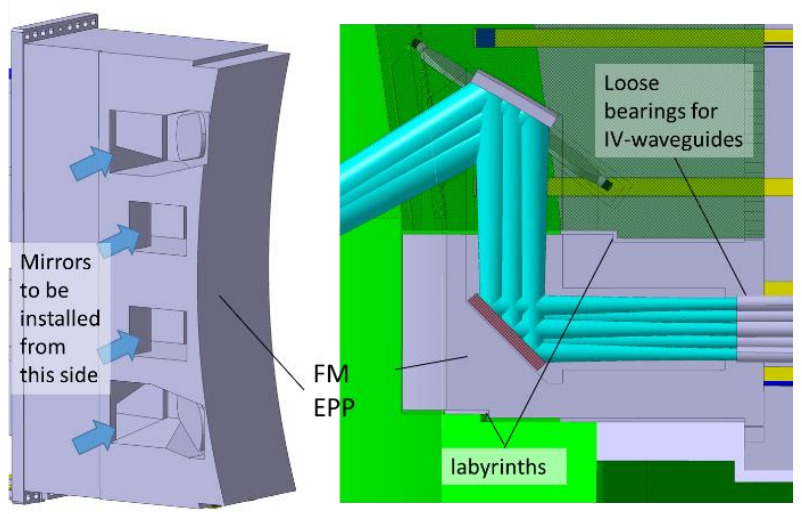

Fig. 8. EC launcher FM EPP (full view and cutaway)

Contrary to the SM EPP the FM EPP has openings for beam propagation and waveguide support also on its rear side. This reduces the shielding capability substantially, which is why additional shielding elements are foreseen to be attached to the rear side of the plug, enclosing the waveguides and the quasi-optical beams, respectively. These auxiliary shields (cf. figure 4) are sketched as simple blocks only in the pre-conceptual model and shall serve as input for the forthcoming MCNP neutronic analysis. More engineering effort will be done if their need for shielding optimisation is approved.

\section{Maintenance Concept}

Preliminary evaluations indicate the installations of the EC launcher as to be maintained either regularly or on purpose during EU DEMO operational lifetime. In order to perform the required procedures in an effective way, the replacement of the plugs is preferred instead of the replacement (or repair) of individual mirrors or other smaller sized components.

The SM EPP and its integration is designed for multiple replacement during DEMO operation, which is why the whole procedure is planned to be slightly simpler than that of the FM EPP.

Due to the dogleg layout of the millimeter wave system, neither In-Vessel nor Ex-Vessel waveguides must be removed for the exchange of the SM EPP. However, a full opening of the confinement system of the port through one single closure plate would counteract this advantageous situation, as the de-installation of such a plate would conflict with the Ex-Vessel millimeter wave system.

Thus a staggered closure plate arrangement has been designed, which allows the removal of the SM EPP through a minimum sized opening without being obstructed by other components in the port cell. To take out the SM EPP the Remote Handling (RH) cask must be applied to the rear side of the closure plate and its subplate (cf. chapter 7) will be un-sealed, loosened and removed. Then customized RH tools will cut the supply lines near the port plug. The supply lines are located at the inner wall of the equatorial port and then guided around the whole closure plate assembly in order to avoid further cutting of these delicate installations. Finally, the port plug will be unbolted and a push-pull tractor will be approached to retract the port plug into the cask. Then it will be moved into the Hot Cell facility for exchange or repair.

For the FM EPP the procedure is more complex. To remove this component, dismantling of the whole ExVessel waveguide system is required. Then, also after applying the RH cask, the closure plate can be opened and (if not yet done through the sub-plate) the SM EPP must be de-installed since the labyrinth layout blocks any free movement of the FM side.

If these preparatory steps have been performed, the supply lines of the FM EPP can be cut and the flange joint of the plug can be loosened. The major maintenance steps (1.1 through 1.4 for the SM EPP and 2.1 through 2.6 for the FM EPP) are illustrated in figure 9.

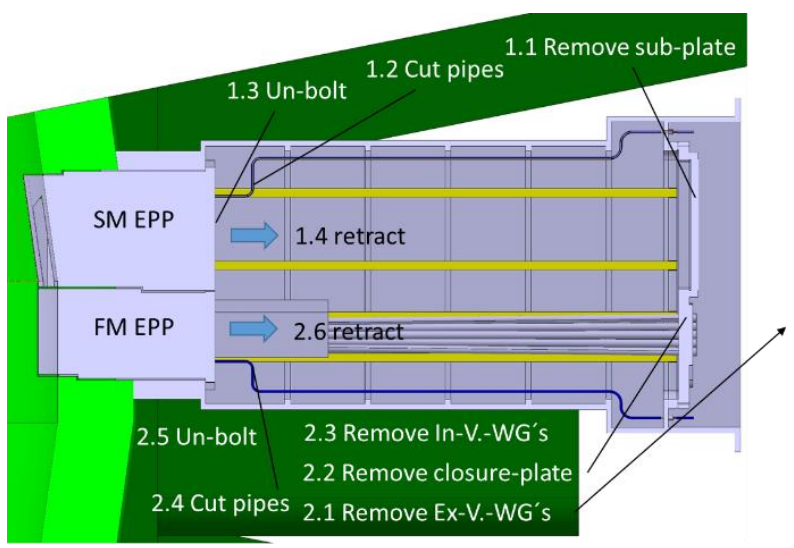

Fig. 9. Major steps for maintenance removal of the EPP's.

\section{Closure Plate assembly}

The maintenance concept described in chapter 6 requires an adequate layout for the accessible vacuum boundary of the equatorial port. Thus, this so-called closure plate has been sub-divided into a staggered assembly, consisting of several elements, to be opened for dedicated purpose.

The main closure plate (1) is formed as a massive plate, which areal dimension is $3,880 \times 2,830 \mathrm{~mm}^{2}$. It has a thickness of $110 \mathrm{~mm}$ and carries all other sub-plates. It is attached to the port extension by bolts and its net weight is $4,520 \mathrm{~kg}$.

The closure plate sub-plate (2) is integrated into the closure plate in order to allow individual removal of the Steering Mirror EPP without removing the waveguide assemblies. Its areal dimension is $3,640 \mathrm{x} 1,530 \mathrm{~mm}^{2}$. It is bolted towards the closure plate. With its thickness of $80 \mathrm{~mm}$, it has a weight of $3,470 \mathrm{~kg}$. 


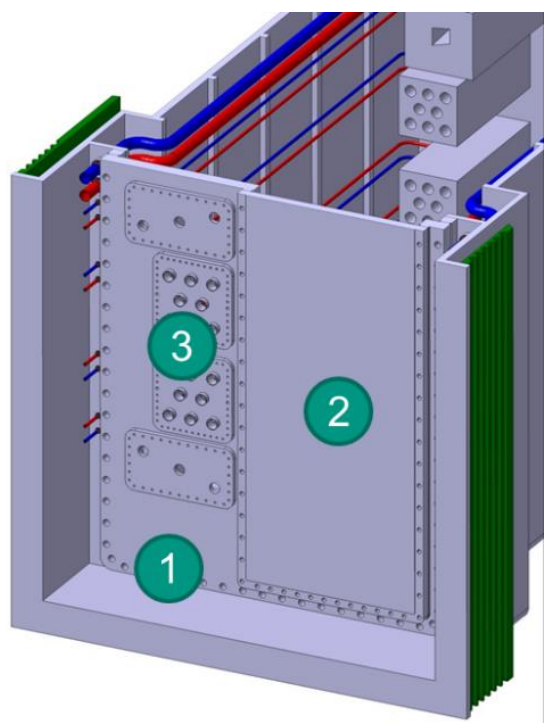

Fig. 10. Closure plate staggered assembly

Ex-Vessel- and In-Vessel waveguides are connected via WG-feed-throughs, gathered on individual subclosure plates (3) for each waveguide section. The pre-conceptual areal dimensions of the sub-plates is $890 \times 485 \mathrm{~mm}^{2}$ for the SM side and $670 \times 650 \mathrm{~mm}^{2}$ for the FM side. With the thickness chosen to be $40 \mathrm{~mm}$, their mass is ca. $125-130 \mathrm{~kg}$ for each.

All closure plates, being part of the First Confinement System (FCS) require a double sealing, which can be monitored. Optional designs can feature DMS (double metallic sealing) or lip-weld sealing. The entire layout of the closure plate assembly is given in figure 10, indicating the numbers of the components, used in chapter 7 .

\section{Neutronic considerations}

In the EU DEMO plant description document [6], neutronic design limits are given regarding neutron damage, Helium production and Shutdown dose rates for several areas and types of components for an EU DEMO reactor.

Since the demonstration of fulfilling these design limits with the pre-conceptual design can be made not before a dedicated MCNP neutronic analysis has been run, a rather conservative approach has been chosen for designing the EC Launcher components. Typical consequences from this approach are the largely oversized port plugs (providing shielding structure of minimum $200 \mathrm{~mm}$ thickness between any open structure and the vacuum vessel welds), relative to the dimensions that would be required to encase the millimeter wave system from plain geometrical aspects. A further development under this approach is the concept of the auxiliary shields. Neutronic calculations are currently in preparation and their results will be incorporated into the next design upgrade.

\section{Versatility of the design}

The present pre-conceptual design of the EC launcher prefers a combination of OEWG with a quasi-optical dogleg beam configuration for plasma heating and a MSA concept for NTM stabilization. However, the current concept of an equatorial port with dedicated port plugs for individual millimeter-wave component assemblies and optional auxiliary shield blocks has the freedom to be adapted easily for further millimeter wave concepts as well. Another important feature, which offers particular design versatility, is the maintenance approach with staggered closure plates and sidestepped supply lines. To implement the possibility of such design changes already in the present CAD model, particular geometries are designed with a skeleton geometry, which allows quick adaptation of the relevant components.

\section{Conclusion}

A EUROfusion task has been placed to advance the preconceptual design for an EU DEMO EC launcher. Starting from the engineering baseline 2017 model, the physics baseline 2018 and a new MSA and OEWG antenna design from ISTP, a design concept has been established and corresponding CAD models were created. Beside proper integration into the baseline models, also RH maintenance schemes and neutronic aspects were taken into account. The model will be the basis for forthcoming MCNP- and EM-analyses.

\section{Acknowledgments}

This work has been carried out within the framework of the EUROfusion Consortium and has received funding from the EURATOM research and training programme 2014-2018 and 2019-2020 under grant agreement No. 633053. The views and opinions expressed herein do not necessarily reflect those of the European Commission.

\section{References}

[1] C. Bachmann: "Overview over DEMO design integration challenges and their impact on component design concepts", Fusion Engineering and Design (2018), https://doi.org/10.1016/j.fusengdes.2017.12.040

[2] S. Garavaglia: "EU DEMO EC equatorial launcher preconceptual performance studies", 2019, Proceedings of this conference

[3] T. Franke et al.: "Initial port integration concept for EC and NB systems in EU DEMO tokamak", Fusion Engineering and Design (2019), https://doi.org/10.1016/j.fusengdes.2019.03.007

[4] C. Gliss: EU DEMO engineering baseline model, CAD assembly, 2018, private communication

[5] W. Young et al.: Roark's formulas for stress and strain, McGraw-Hill, $8^{\text {th }}$ edition, 2012

[6] C. Bachmann: EU DEMO Plant description document, release $1.5,2019$, private communication 